\title{
Ethnologies
}

\section{L’expérience migratoire et le sentiment d'appartenance}

\section{Lucille Guilbert}

Volume 27, numéro 1, 2005

Appartenances

Belonging

URI : https://id.erudit.org/iderudit/014020ar

DOI : https://doi.org/10.7202/014020ar

Aller au sommaire du numéro

Éditeur(s)

Association Canadienne d'Ethnologie et de Folklore

ISSN

1481-5974 (imprimé)

1708-0401 (numérique)

Découvrir la revue

Citer ce document

Guilbert, L. (2005). L'expérience migratoire et le sentiment d'appartenance.

Ethnologies, 27(1), 5-32. https://doi.org/10.7202/014020ar

Ce document est protégé par la loi sur le droit d'auteur. L'utilisation des services d'Érudit (y compris la reproduction) est assujettie à sa politique d'utilisation que vous pouvez consulter en ligne.

https://apropos.erudit.org/fr/usagers/politique-dutilisation/ 


\section{L'EXPÉRIENCE MIGRATOIRE ET LE SENTIMENT D’APPARTENANCE}

\section{Lucille Guilbert \\ Université Laval}

L'histoire de l'humanité est une histoire des migrations. Par ailleurs, on assiste à une complexification et à une diversification des trajectoires migratoires. Les catégorisations habituelles en types de migration migration volontaire, migration forcée, migration économique fondées sur les motivations en termes de facteurs de départ [push factors] et de facteurs d'attraction [pull factors] se conjuguent en des configurations flottantes chez un même individu ou un même groupe. Le modèle univoque de la migration d'un point d'origine à un point d'arrivée correspond peu aux situations contemporaines. La migration est un déplacement multidirectionnel et, comme le disent Droz et Sottas, « la conception d'un déménagement définitif ou d'un déplacement irréversible ne rend pas compte de la réalité sociale, car les phénomènes migratoires observables aujourd'hui sont — pour la plupart bidirectionnels ou circulaires » (1997: 70). Le plus souvent, les trajectoires migratoires sont ponctuées d'aller-retour, de va-et-vient fréquents entre divers lieux : pays natal ou pays de résidence, pays où sont installés des membres de la famille dispersée ou la collectivité en diaspora, pays d'adoption. Cette mobilité entraîne des réaménagements des identifications culturelles et sociales et des appartenances à des groupes ou à des réseaux locaux, nationaux ou transnationaux.

Les problématiques prises en compte par l'ethnologie des migrations marquent une distanciation épistémologique avec l'anthropologie classique car elle rompt avec la conception harmonieuse de «l'interdépendance étroite milieu-environnement-société ». Dans ses analyses des immigrants et des réfugiés, des communautés diasporiques 
et des communautés transnationales, l'ethnologue Pierre Centlivres démontre que «si l'idée de sociétés vivant en rapport étroit avec leur environnement naturel et humain était juste, croire que c'est un rapport immémorial et intangible est faux » (Amiotte-Suchet et Floux 2002 : 9). Par exemple, les observations de Pierre Centlivres et Micheline Centlivres-Demont (2000) dans les camps de réfugiés du Pakistan ont révélé que les Afghans et les Turkmènes qui s'y trouvaient avaient été réfugiés plusieurs fois auparavant. Alessandro Monsutti approfondit cette perspective dans ses recherches sur les réseaux sociaux et les stratégies économiques des Hazaras d'Afghanistan. Il montre que le fait de se déplacer «pour chercher des emplois, pour échapper à une sécheresse ou fuir une guerre est une expérience commune en Afghanistan» (2004 : 54). Monsutti démontre que ces migrations ne dissolvent pas les appartenances communautaires ; souvent, elles les intensifient plutôt et les diversifient au fil des lieux traversés au point que ces appartenances constituent des ressources socioculturelles qui mobilisent la circulation d'information, d'argent et d'influences.

Qu'elle soit volontaire en vue de la réalisation de projets personnels et promotionnels ou qu'elle soit forcée par des catastrophes naturelles ou par des conflits au sein du pays de départ, la migration entraîne le relâchement ou la rupture de certains liens sociaux affectifs et professionnels et la perte de repères géographiques, sociaux et culturels. Cette même migration suscite par ailleurs une construction de nouvelles alliances et rapports à l'Autre, une appropriation de nouveaux lieux physiques et symboliques. Une élaboration identitaire se profile en permanence et questionne les appartenances (Camilleri et Vinsonneau 1996 : 68-69 ; Vinsonneau 2002). Mucchielli définit l'appartenance comme un processus qui «implique une identification personnelle par référence au groupe (identité sociale), des attaches affectives, l'adoption de ses valeurs, de ses normes, de ses habitudes, le sentiment de solidarité avec ceux qui en font aussi partie, leur considération sympathique » (1980 : 99). Mais à l'ère de "l'homme flexible », pour reprendre l'expression de Frédéric de Coninck, où l'appartenance à une famille, un groupe de travail, une communauté, une nation, devient plus floue, moins durable et multiforme et est marquée par l'élargissement des réseaux sociaux, le sentiment d'appartenance se construit davantage dans une trajectoire individuelle de stratégies et de choix personnels (Coninck 2001 : 7-81). On peut définir le sentiment d'appartenance comme l'émotion de se considérer comme partie intégrante d'une 
famille, d'un groupe ou d'un réseau. Ce qui n'exclut pas que ce choix converge et englobe les membres d'une famille ou d'un groupe. En ce sens, Michèle Vatz-Laaroussi (2001), sur les stratégies familiales des familles immigrantes, montre la force du sentiment d'appartenance en action à travers les stratégies familiales d'adaptation à leur société d'adoption. De plus, le sentiment d'appartenance varie avec les traditions historiques et culturelles qui ont intégré les migrations et la mobilité comme stratégie de survie et stratégie de développement. Il est modulé aussi par la personnalité et les projets individuels et familiaux du migrant. À ce titre, les exemples fournis par Centlivres et Centlivres-Demont (2000) et par Monsutti sur les réfugiés afghans nous conduisent à réviser certaines positions. Même dans des situations objectives et difficiles de migrations forcées, la migration peut comporter une dimension affective positive, le sentiment d'accomplir un rite d'initiation vers l'âge adulte et acquérir un plus sur le marché matrimonial.

Il a été demandé aux auteurs d'Appartenances/ Migrations/Belonging, de s'interroger sur les significations attribuées au processus migratoire par les acteurs migrants, sur le développement de sentiments d'appartenance à travers et à partir de l'expérience migratoire. La pluralité des différents ancrages disciplinaires et multidisciplinaires, des angles d'observation, des questionnements épistémologiques, théoriques et méthodologiques, des pratiques professionnelles, apportent un éclairage nouveau sur l'inventivité des pratiques culturelles et sur la formation des réseaux d'appartenances nationaux et transnationaux. Au-delà des représentations idéologiques des lieux et des personnes, on observe des renouvellements et des "recadrages " méthodologiques qui permettent des conceptualisations fondatrices de nouveaux rapports à l'Autre. Certains auteurs explorent davantage les pratiques culturelles et sociales dans l'expérience de réfugié et de personne déplacée de même que l'élaboration des réseaux locaux, nationaux et transnationaux (Monssutti, Dicum, Mosquera-Labbé, Morissette). D'autres se concentrent davantage sur les dimensions symboliques et émotionnelle du sentiment d'appartenance en regard de la transmission de la mémoire migrante, du projet identitaire, de la transmission intergénérationnelle et débouchent sur des pratiques de médiation culturelle (Meintel et Kahn, Dorais, Boucher, Guzin-Lukic). D'autres encore examinent les rapports à des situations plus en marge des phénomènes migratoires tels qu'on les conçoit ordinairement. Une analyse des dynamiques identitaires et le sentiment d'appartenance de jeunes professionnels dans 
la fonction publique d'un État officiellement multiculturel et multilingue enrichit la réflexion sur les phénomènes similaires en situation de migration (Veillette). Les voyages touristiques de retour au pays d'origine (Fourcade) et les voyages de longue durée unissant loisirs et travail dans une forme de vie nomade moderne, le full-time RVing (Forget) illustrent quelques-unes des formes de mobilité qu'il reste à circonscrire.

La contribution d'Alessandro Monsutti pose une réflexion épistémologique sur les méthodologies de terrain et les méthodes d'investigation. Au lieu de se fixer un lieu d'observation fixe, l'observation in situ, l'ethnologue va davantage suivre le mouvement des individus et des groupes dans leurs pérégrinations. Pour effectuer l'étude des réseaux, l'ethnologue ne se contentera plus de reproduire les relations par des graphes, mais suivra l'élaboration de ses réseaux. L'ethnologue se fait lui-même migrant. Monsutti utilise une méthodologie d'étude des réseaux renouvelée et articulée autour des relations interpersonnelles et des déplacements afin d'examiner les pratiques culturelles et sociales, les stratégies de diversification économique et de survie à travers la dispersion spatiale. Il en résulte que la distinction entre les catégories de réfugié, de personne déplacée, de migrant volontaire, de migrant économique est ici très nuancée car une large proportion de cette population hazaras présente, simultanément ou successivement, des traits de toutes ces catégories. Ces réfugiés ne sont pas de simples victimes d'un sort qui les dépasse, dit Monsutti, ils savent utiliser leurs ressources culturelles pour s'adapter à des circonstances dramatiques, ils développent un certain nombre de stratégies sociales similaires à celle des migrants économiques et un individu peut appartenir à plusieurs catégories à la fois ou passer de l'une à l'autre au cours de son existence. Au cours de ces va-et-vient fréquents, « il ne s'agit dès lors pas d'une rupture des liens sociaux, puisque ces déplacements s'insèrent dans la représentation de mobilité que les gens se font du parcours de la vie d'un homme». Le sentiment d'exil et le sentiment d'appartenance s'ancrent dans les représentations du déplacement, des significations que l'on attribue à ces déplacements et de l'utilisation qu'on en fait. Les travaux d'Alessandro Monsutti sur les réseaux afghans hazaras révèlent l'appropriation de l'expérience de réfugié et de migration en terme de «principe de vie » (2004: 185) et comment la migration est utilisée comme stratégie économique à travers des réseaux de solidarité et d'entraide. 
Les réseaux sociaux que tissent les migrants au cours de leur migration ou de leur déplacement forcé au sein de leur pays ou ailleurs leur offrent des opportunités de découvrir leurs droits et de nouvelles ressources pour faire reconnaître ces droits en participant à des associations solidaires. Claudia Mosquera-Labbé montre, à travers le récit de quinze femmes afro-colombiennes déplacées, la forte précarité de la situation de la personne déplacée qui ne reçoit pas les protections accordées aux personnes réfugiées. Par ailleurs, les analyses de MosqueraLabbé dévoilent les nouvelles pratiques sociales de ces femmes déplacées qui découvrent qu'elles sont des sujets de droit et qu'elles peuvent revendiquer leurs droits à travers des pratiques associatives en se transformant ainsi en sujets politiques.

Les migrations confrontent non seulement les pratiques culturelles et sociales des migrants mais tout autant les pratiques professionnelles des intervenants sociaux et des éducateurs auprès des populations réfugiées ou déplacées.

Julia Dicum présente un bilan théorique sur les programmes d'éducation en situation de crise en regard d'une expérience réalisée auprès de jeunes réfugiés afghans dans des camps de réfugiés pakistanais. Le séjour dans un camp de réfugiés constitue pour ces jeunes afghans à la fois un lieu d'acculturation et un lieu d'expériences sociales et politiques. L'éducation en situation d'urgence dans l'espace culturel de camp de réfugiés, en situation de transition, nécessite un équilibre entre une orientation à la communauté et un espace d'apprentissage et d'expériences nouvelles.

Le sentiment d'appartenance d'un individu à une collectivité se développe de pair avec la capacité qu'il a de pouvoir exprimer ses besoins, ses attentes, ses suggestions et le sentiment d'être reconnu dans la contribution qu'il peut apporter quelque chose à cette société (Guilbert 2004 ; 2005a). Pour une intégration réussie des nouveaux arrivants, une société se doit de mettre en œuvre des accommodements raisonnables, des passerelles, des médiations efficaces. Une bonne maîtrise de la langue de pays d'accueil constitue un atout incontournable d'une intégration réussie et cette maîtrise s'acquiert progressivement. Le recours à un interprète est un droit identitaire fondamental et s'avère indispensable au moment de l'accueil du nouvel arrivant et au cours des premières années d'installation, en des occasions de moins en moins fréquentes au fur et à mesure que les efforts conjugués du nouvel arrivant et des mesures d'accueil et d'accompagnement produisent leurs fruits. 
Le contexte de situation dans lequel la relation du nouvel arrivant avec son environnement social est facilitée par l'action d'une personne qui réalise une médiation de langage varie considérablement selon que l'action se produit dans la sphère semi-privée, publique ou institutionnalisée. La sphère publique elle-même nécessite différents styles d'interprétariat selon les exigences de la situation ; les attentes face à un interprète en contexte juridique ou lors de la visite chez un médecin ou autre service social ne répondent pas aux mêmes critères. En effet, les fonctions attribuées à un interprète varient selon les contextes d'intervention et selon les cultures d'intervention et du rapport de force qui s'y déroule (Roy et Kapoor-Kohli 2001 ; Guilbert 2002a ; 2002b). Tant lors de démarches de médiation citoyenne interculturelle que dans les ateliers de formation mutuelle (Guilbert 2005), nous avons été confrontés à une vive polémique concernant les attentes face aux interprètes. Certains, souvent des gestionnaires de banques d'interprètes ou de services, préconisaient le mot à mot fidèle lors des interactions et l'effacement physique, culturel, expérientiel de la part de l'interprète, en justifiant cette exigence par une compréhension assez singulière de la notion de neutralité. D'autres participants, souvent des réfugiés, revendiquaient plusieurs changements dans la gestion des services d'interprétariat. Ils préconisaient une plus grande disponibilité des horaires de prestations de services d'interprétariat, notamment des services d'urgence sept jours par semaine; le droit d'avoir le choix de l'interprète qui accompagnera, le droit de refuser un interprète en qui on n'a pas confiance ou qui par sa présence même offenserait l'intimité des propos; une compréhension du rôle de l'interprète qui dépasse le mot à mot pour rejoindre davantage une fonction d'explication et de médiation culturelle. D'autres encore, souvent des interprètes, reproduisaient dans leurs propos le discours officiel de leurs employeurs concernant le mot à mot et l'effacement des interprètes alors que l'observation de leurs pratiques dévoilait une approche sensible des besoins et des situations ; souvent, sans le conceptualiser, l'interprète tenait un rôle de passeur, de médiateur interculturel auprès des porteurs de cultures différentes.

Karine Morissette examine la trajectoire migratoire et professionnelle d'immigrants interprètes dans la région de Québec. Elle cerne différents profils d'interprètes, professionnels dans la sphère publique, «spontanés » dans la sphère privée, et discute de différentes conceptions quant au rôle de l'interprète. La tension linguistique dans le contexte 
québécois francophone et les enjeux démographiques du Québec au sein du Canada majoritairement anglophone influencent-ils la pratique professionnelle? Le rôle de médiation interculturelle peut-il véritablement fonctionner au sein d'un acte professionnel, voire médical, administratif, législatif? La triade formée par l'allophone, l'interprète et l'intervenant peut-elle s'échapper d'un rapport de force?

Le sentiment d'appartenance s'inscrit dans des logiques sociales et culturelles. Il se construit à travers des dimensions à la fois symboliques et émotionnelles fortes. La question d'appartenance renvoie inéluctablement à la notion d'identité. Loin des courants qui conceptualisaient l'identité en un cumul de caractéristiques innées transmises biologiquement, les courants actuels, marqués par les dynamiques des migrations internationales et par l'emprise des phénomènes de mondialisation, reconnaissent la malléabilité et la mouvance de la notion d'identité. La réflexivité et l'idéologie pluraliste interviennent dans ce travail définitoire de soi-même. Deirdre Meintel et Emmanuel Kahn montrent que le sentiment d'appartenance peut s'inscrire également dans un projet identitaire plus conscient et volontariste de la part des parents, plus diffus et constructiviste de la part des enfants. Alors que les parents projettent leur aspiration identitaire sur leurs enfants dans le futur, les enfants tissent leur identité au présent au gré des opportunités et des choix spontanés. Lorsqu'ils sont eux-mêmes parents, ils conjuguent ce qu'ils ont acquis de leurs parents et les enjeux qui les interpellent dans la réalité pluraliste de la société québécoise dans le contexte de la mondialisation contemporaine. Ce projet peut donc prendre la forme d'une négociation intergénérationnelle. Cette dimension est prise en charge également dans les recherches de Dorais et de Boucher. Ainsi, l'appartenance ethnique relève d'un sentiment d'identité qui se développe constamment; elle n'est plus conçue comme une caractéristique innée, même si, précisent Meintel et Kahn, "dans l'expérience subjective des personnes, ce sentiment persiste encore ». Tout comme Monsutti le fait pour saisir les situations de mobilité spatiale et sociale, Meintel et Kahn revisitent la méthodologie de l'étude des réseaux pour arriver à une meilleure compréhension de la société "comme un ensemble de relations sociales ramifiées à partir de chaque individu ».

La construction du sentiment d'appartenance à une collectivité est influencée par le rapport majoritaire-minoritaire non seulement en contexte de migration, mais également dans le contexte des sociétés 
fédérales qui promeuvent des politiques de multiculturalisme et de multilinguisme. Josianne Veillette analyse la construction identitaire de jeunes travailleurs québécois et suisses dans la fonction fédérale de leur pays respectif. Sans être engagés dans une expérience migratoire, ces jeunes professionnels sont en situation quotidienne de négocier leur identité culturelle et ethnique et leur identité professionnelle à travers leurs stratégies et positionnement face à leur capacité et à leur choix d'utiliser l'une ou l'autre des langues officielles selon les contextes de travail et l'équilibre des jeux de pouvoir. À la fois, les cultures institutionnelles et les dynamiques de groupes influencent fortement le développement d'un sentiment d'appartenance tant dans l'expérience migratoire que dans l'environnement de travail dans un milieu plurilingue et multiculturel.

Une société peut favoriser le développement du sentiment d'appartenance de ses membres à la collectivité à travers des pratiques de médiation culturelle dans l'espace public (Lamizet 1999; Guilbert 2004). Les contributions de Louis-Jacques Dorais, de Colette Boucher et de Nada Guzin-Lukic explorent diverses facettes de médiations culturelles par l'oralité, l'écriture et l'espace muséal.

À partir d'un outil de sensibilisation au pluralisme culturel, réalisé grâce à l'étroite collaboration d'un anthropologue (Dorais 2003) et d'aînées et aînés vietnamiens, Dorais explique comment le récit de vie d'immigrés ouvre une fenêtre sur la manière dont l'identité culturelle se construit et est remémorée. Les souvenirs d'enfance, l'identité culturelle vietnamienne à la fois conservée, transformée, fantasmée, deviennent une ressource sociale à partager avec la jeune génération d'enfants nés au Québec d'immigrants vietnamiens et avec l'ensemble de la population québécoise. L'écriture ethnographique choisie par Dorais déploie une mise en scène fictive d'une discussion d'aînées et d'aînés autour d'un thé chez le Dr Quynh, personnage légendaire de la tradition orale vietnamienne. La mise en scène fictive rappelle les procédés littéraires dans la tradition des Évangiles des quenouilles, de Boccace, de Marguerite de Navarre. Toutefois, elle sert ici d'abord le projet des acteurs migrants eux-mêmes. L'ethnologue accepte le rôle instrumental de soutien, de catalyseur et de canal de transmission auprès d'acteurs sociaux engagés qui élaborent et mènent à terme leur entreprise culturelle. Cette médiation culturelle se réalise. Le sentiment d'appartenance au pays d'origine et le sentiment d'appartenance au pays d'immigration ne sont pas en concurrence ni en opposition. Une 
voie s'ouvre : le développement d'un sentiment d'appartenance au pays d'adoption est tributaire de la libre expression et de la reconnaissance du sentiment d'appartenance au pays d'origine et au pays d'adoption.

Le déploiement de ce sentiment d'appartenance à deux ou plusieurs entités culturelles et nationale se construit dans un espace transnational. Monsutti décrit le cas afghan. Dorais a exploré ailleurs ces identités transnationales chez les Vietnamiens $(2004 ; 1998)$. Les récits de vie analysés par Monsutti, Meintel, Dorais et Boucher font écho à cette transmission de la mémoire familiale et à ce désir de partager un sentiment d'appartenance. L'entrevue réalisée avec l'auteur québécoise d'origine haïtienne Marie-Célie Agnant par Colette Boucher pose les questions de l'oralité et de la transmission des valeurs culturelles par l'intermédiaire du récit oral investi et célébré dans le récit littéraire. L'écriture littéraire opère une médiation culturelle par laquelle les pratiques langagières, le français et le créole, le sentiment d'appartenance au pays d'origine et au pays d'adoption, la communication intergénérationnelle se transforment et s'interpénètrent en créant des configurations identitaires innovantes et ouvertes sur un enrichissement constant. Ces réinterprétations et reconfigurations des sentiments d'appartenance se font toutefois à la manière d'une lutte avec soi-même sinon avec les autres. L'image de la guerrière évoque cet esprit de lutte, de courage et d'intégrité qu'il faut à tout prix assurer pour soi et pour ses descendants. Le personnage de la mère, plus particulièrement de la grand-mère, tient le rôle de médiatrice dans cette transmission du patrimoine culturel et linguistique.

Le sentiment d'appartenance à une collectivité se construit d'une manière privilégiée dans l'espace public (Lamizet 1999). L'institution muséale peut offrir une médiation artistique susceptible de nourrir les identifications à une collectivité, de réduire la fracture sociale par la production d'un «lien social» (Caune 1999: 44). Amorçant un prolongement à ses travaux sur la médiation muséale en contexte de réconciliation après les conflits survenus en Bosnie et Herzégovine (Guzin-Lukic 2004 ; 2005), Nada Guzin-Lukic élargit sa réflexion à la médiation culturelle du musée au sein de la société pluraliste québécoise. Les musées peuvent être le lieu d'une médiation entre le citoyen et la ville, la région ou le pays. Les Québécois natifs et les Québécois issus de l'immigration réagissent-ils de la même manière devant une même exposition sur l'évolution de la culture québécoise ? Le discours politique 
et social véhiculé par le Musée donne-il une juste représentation de la réalité plurielle du Québec d'aujourd'hui?

La quête de souvenirs et le désir de transmission culturelle dans le pays d'adoption traversent aussi la contribution de Marie-Blanche Fourcade. Fourcade décrit les différents aspects des rapports entre souvenirs touristiques et diaspora. Des immigrants arméniens au Québec font plus ou moins régulièrement des voyages de visites dans leur pays d'origine et en rapportent dans leur lieu de vie au Québec des souvenirs touristiques. Ce «tourisme de retour», comme l'appelle Fourcade, présente des caractéristiques différentes du tourisme ordinaire. Ces objets sont-ils vraiment rattachés à une identité arménienne et sont-ils destinés aux mêmes usages qu'auparavant ou sont-ils plutôt des substituts de repères culturels et géographiques perdus ? Dans tous les cas cependant, ces objets constituent de toute évidence pour leur acquéreur un moyen d'exprimer un sentiment d'appartenance à la collectivité arménienne dans son histoire de longue durée et dans la diaspora.

Le foisonnement des formes de mobilité dans le monde actuel metil en danger la capacité de créer des liens sociaux signifiants et d'entretenir un sentiment d'appartenance dans la durée ? L'homme flexible est-il un homme sans attache ? Le mode de vie du "camping à temps plein » illustre une forme extrême de la quête d'une liberté et d'une mobilité sans contraintes. Célia Forget esquisse l'analyse d'un mode de vie mobile, le camping à plein temps ou "full time RVing ", une forme de nomadisme qui allie l'aventure et la liberté au confort nord-américain par le truchement de la technologie de la mobilité et des communications. Comment ces personnes qui ont tout quitté pour vivre dans leur véhicule récréatif et sillonner les routes nord-américaines cultivent-elles un sentiment d'être chez soi tout en se déplaçant? Pour ces «nouveaux nomades », qui ne sont ni des immigrants ni des réfugiés ni des personnes déplacées, la mobilité permanente ne remet en cause ni leur citoyenneté, ni leur sentiment d'appartenance à une nation. La forme de vacances temporaire qu'ils adoptent s'inscrit dans une durée indéterminée et les vacanciers se projettent dans un imaginaire et une vie fantasmée d'itinérant, de gitans et de hobos sans en vivre toutes les implications concrètes. Entre la culture de la mobilité et la volonté d'enracinement, il semble qu'ils aient canalisé ces deux tendances dans une tendance nord-américaine, celle du fantasme du plaisir et de liberté sans contrainte. Le véhicule récréatif devient le symbole de l'imaginaire de la route et d'un mode de vie hédoniste. Les liens sociaux semblent 
toutefois distendus. Les liens familiaux résistent encore, mais uniquement dans la mesure où chaque membre de la famille accepte une réinterprétation, sinon une inversion des rôles de chacun au sein du groupe familial. En effet, à l'exception des liens familiaux, le full time RVing permet-il le développement de vrais réseaux sociaux intenses?

\section{La migration : une opportunité d'apprentissage}

L'ensemble de ces contributions est traversé par des lignes de force convergentes. La prolifération des formes de mobilité actuelles nécessite un examen des typologies de migration et une flexibilité dans l'utilisation de ces définitions pour une compréhension fine de ces expériences humaines diversifiées. L'observation participante à travers la mobilité même du chercheur, les récits de vie des migrants, l'étude des réseaux recentrée sur les dynamiques relationnelles concrètes et les significations tant cognitives qu'émotionnelles que leur attribuent les acteurs sociaux constituent des voies méthodologiques prometteuses. Le sentiment d'appartenance tisse un champ social cohérent malgré la dispersion spatiale. Les situations vécues lors de migrations offrent l'opportunité d'expériences d'apprentissage, d'acquisition de savoirs et de savoir-faire, de développement de compétence, de déploiement de ressources personnelles et sociales.

Les migrations volontaires, mais aussi les migrations forcées, sont l'occasion de nouveaux apprentissages (Guilbert 2005a ; 2005b ; 2005c). La migration forcée est un déplacement marqué par les pertes, les deuils, les souffrances. Paradoxalement, ce voyage douloureux conduit à des expériences d'apprentissage. Les personnes qui ont été jetées dans l'exil ont réalisé, même dans des conditions difficiles, des apprentissages multiples.

On n'a jamais interrogé la possibilité qu'un obstacle à l'intégration de la personne réfugiée ou immigrante soit le déni des apprentissages et des savoirs acquis au cours de la migration. Ces apprentissages peuvent se produire à une étape de la trajectoire migratoire tant au niveau local ou national, qu'international. Ces expériences d'apprentissage peuvent être informelles: les compétences culturelles et leur transformation en compétences professionnelles; la créativité des réponses culturelles aux situations nouvelles de la vie quotidienne; les compétences narratives; l'établissement de réseaux; et la recherche de l'information. Ces expériences d'apprentissage peuvent être formelles, organisées par des 
organismes humanitaires internationaux ou locaux, sous formes de stages, de cours de formation de métiers, de cours de langue. Il faut considérer aussi les expériences de travail (interprète, enseignant de langue, éducateur, assistant travailleur social, commerçant) dans un contexte interculturel et international dans les camps de réfugiés et à toutes les étapes de la migration. Les expériences d'apprentissage sont influencées par plusieurs facteurs tels que les types de camps de réfugiés et leur localisation, l'âge, le genre, le niveau d'instruction, le groupe ethnoculturel auquel s'identifie le réfugié et le groupe ethnoculturel auquel il est identifié.

Il est souhaitable que des études sur les diverses formes de mobilité se multiplient dans une perspective comparative afin de mieux comprendre les liens possibles entre les expériences d'apprentissage, l'impact de la reconnaissance ou la non-reconnaissance de ces expériences d'apprentissage sur l'insertion sociale dans la société d'accueil en termes de résilience, d'obstacle, d'adaptation, de facteurs d'employabilité et de participation sociale et citoyenne à la société d'accueil. 


\section{Références}

Amiotte-Suchet, Laurent et Pierre Floux, 2002, "Voyage avec Pierre Centlivres, de l'Afghanistan aux communautés transnationales ». Ethnographiques.org 1 [en ligne] http://www.ethnographiques.org/ documents/articles/centlivres.html

Camilleri, Carmel et Geneviève Vinsonneau, 1996, Psychologie et culture. Concepts et méthodes. Paris, Armand Colin.

Caune, Jean, 1999, Pour une éthique de la médiation. Le sens des pratiques culturelles. Grenoble, Presses universitaires de Grenoble.

Centlivres, Pierre et Micheline Centlivres-Demont, 2000, «Exil et diaspora afghane en Suisse et en Europe ». Cahiers d'études sur la Méditerrannée orientale et le monde turco-iranien 30 : 151-172.

Coninck, Frédéric de, 2001, L'homme flexible et ses appartenances. Paris et Montréal, L'Harmattan.

Dorais, Louis-Jacques, 2004, "La construction de l'Identité ». Dans Denise Deshaies et Diane Vincent (dir.), Discours et constructions identitaires, Québec, Les Pressses de l'Université Laval : 1-11.

2003, Un thé chez le docteur Quynh. Sept aînés vietnamiens parlent de leur culture. Québec, Association Inuksiutiit Katimajiit.

Dorais, Louis-Jacques, 1998, Identités transnationales chez les Vietnamiens du Québec. Département d'anthropologie, Université Laval.

Droz, Yvan et Beat Sottas, 1997, "Partir ou rester ? Partir et rester. Migrations des Kikuyu au Kenya ». L'Homme 142 : 69-88.

Guilbert, Lucille, à paraître, «La Ville de Québec. Un laboratoire pour la mise en œuvre de médiations avec les réfugiés ». Revue Études ethniques au Canada / Canadian Ethnic Studies Journal XXXVII (3). -, 2005b, «Les expériences d'apprentissage et la construction des savoirs au cours de la migration ». Dixième Congrès de l'Association internationale de Recherche interculturelle (ARIC), Alger, 1-8 mai. -, 2005c, "Knowledge and learning experiences acquired by refugees ». Summer Course on Refugee Issues, Centre for Refugee Studies, Toronto, June 18.

, 2004, "Médiation citoyenne interculturelle. L'accueil des réfugiés dans la région de Québec ». Dans Lucille Guilbert (dir.), Médiations et francophonie interculturelle, Québec, Les Presses de l'Université Laval : 199-220.

-, 2002a, "L'interprète dans sa communauté : un pont vivant ». Session de Formation des interprètes, Québec, Centre international des femmes. 
— - 2002b, «Au cœur des identités, l'interprète ». Communication présentée au Congrès ACFAS, Québec, Université Laval.

Guzin-Lukic, Nada, 2004, "Patrimoine, musée et médiation». Dans Lucille Guilbert (dir.), Médiations et francophonie interculturelle, Québec, Les Presses de l'Université Laval : 139-157.

_- 2004, "Les musées et la guerre en ex-Yougoslavie. Vers une muséologie de la réconciliation ». Université Laval, thèse de doctorat.

Lamizet, Bernard, 1999. La médiation culturelle. Paris, L'Harmattan.

Monsutti, Alessandro, 2004, Guerres et migrations. Réseaux sociaux et stratégies économiques des Hazaras d'Afghanistan. Neuchâtel et Paris, Éditions de l'Institut d'ethnologie/ Éditions de la Maison des sciences de l'homme.

Mucchielli, Roger, 1980, Le travail en groupe. Issy-les-Moulineaux, Éditions ESF.

Vatz Laaroussi, Michèle, 2001, Le familial au cour de l'immigration. Les stratégies de citoyenneté des familles immigrantes au Québec et en France. Montréal, L'Harmattan.

Vinsonneau, Geneviève, 2002, L'identité culturelle. Paris, Armand Colin. 


\title{
The Migratory ExPerience AND the Feeling of BELONGING
}

\author{
Lucille Guilbert \\ Université Laval
}

Human history is a history of migrations. Furthermore, today's migratory trajectories are becoming more complex and diversified, as interested observers can attest. The usual categorizations for types of migrations - voluntary migration, forced migration and economic migration - based on motivations related to push factors and pull factors tend to combine in floating configurations for the same individual or group. The all-encompassing model of migration as a trajectory from a point of origin to a point of arrival is of little use in describing contemporary situations. Migration involves multidirectional displacement, and as Droz and Sottas contend, "the notion of a definitive move or irreversible displacement does not take into account social reality, for the migratory phenomena observable today are - for the most part - bi-directional or circular" (1997: 70). More often than not, migratory trajectories are punctuated by return trips, frequent backand-forth movements between various places: for instance, between one's country of birth and his or her country of residence, and/or between the country where members of one's dispersed family have settled, or the diaspora community and one's country of adoption. This mobility leads to a re-designing of cultural and social identifications and group memberships, as well as of local, national and transnational networks.

The issues taken into account by ethnology concerning migrations reflect an epistemological distancing with respect to classical anthropology by breaking with the harmonious conception of "the close interdependence between community, environment and society". In 
his analyses of immigrants and refugees, of diaspora and transnational communities, the ethnologist Pierre Centlivres shows that "even if the notion of societies living in a close relationship with their natural and human environments is fundamentally correct, it is a mistake to believe that this is an inviolable relationship dating from time immemorial" (Amiotte-Suchet and Floux 2002: 9). For instance, the observations of Pierre Centlivres and Micheline Centlivres-Demont (2000) in the refugee camps of Pakistan have demonstrated that the Afghans and Turkmen found there had already been refugees a number of times before. Alessandro Monsutti broadens this perspective in his research concerning the social networks and economic strategies of the Hazaras of Afghanistan. He shows that "[the] fact of moving in order to search for jobs, to escape drought or to flee from war is a commonplace experience in Afghanistan" (2004: 54). Monsutti demonstrates that these migrations do not dissolve the bonds of community membership; instead they often serve to strengthen and diversify them as more and more places are crossed, to the point where these membership bonds constitute sociocultural resources that mobilize the circulation of information, money and influence.

Whether voluntary, with a view to carrying out personal or special projects, or forced by natural catastrophes or conflicts within the country of departure, migration brings about a weakening or rupture of certain social, emotional and professional bonds and the loss of geographical, social and cultural points of reference. Furthermore, this same migration gives rise to the construction of new alliances and relationships with the Other, to an appropriation of new physical and symbolical spaces. Identity is constantly being outlined and developed, and this brings all forms of belonging into question (Camillerri and Vinsonneau 1996: 68-69; Vinsonneau 2002). Mucchielli defines belonging as a process that "involves personal identification with reference to the group (social identity), emotional attachments, the adoption of the group's values, norms and habits, and a feeling of solidarity with those who are also members, their sympathetic consideration" (1980: 99). But in this era of "the flexible human being" (to borrow Frédéric de Coninck's expression), where belonging to a family, a work group, a community, and/or a nation has become many-sided as well as less clearly defined and more ephemeral and is characterized by the expansion of social networks, the feeling of belonging is increasingly constructed within an individual trajectory of personal choices and strategies (De Coninck 
2001: 7-81). The feeling of belonging can be defined as the sentiment of considering oneself an integral part of a family, group or network, not excluding the fact that this choice may converge and encompass the members of a family or group. In this sense, concerning familial strategies of immigrant families, Michèle Vatz-Laaroussi (2001) demonstrates the strength of the feeling of belonging in action through familial strategies of adapting to their society of adoption. In addition, the feeling of belonging varies according to the historical and cultural traditions that have integrated migrations and mobility as strategies of survival and development. It is also modulated by the personality as well as by the individual and family projects of the migrant. In this regard, the examples provided by Centlivres and Centlivres-Demont (2000) and by Monsutti concerning Afghan refugees lead us to reappraise certain positions. Even when forced, i.e. in situations which are objectively difficult, migration may include a positive emotional dimension as it comes to represent the completing of an initiation rite into adulthood and the acquiring of "added value" on the matrimonial market.

The authors of this special issue, Appartenances/ Migrations/Belonging have been asked to ponder the meanings attributed to the migratory process by the migrant players themselves, as well as the development of feelings of belonging through and based on the migratory experience. The many and varied disciplinary and multi-disciplinary anchorings, angles of observation, epistemological, theoretical and methodological interrogations, and professional practices shed new light on the inventiveness of cultural practices and on the development of national and transnational networks of belonging. Above and beyond ideological representations of places and people, one can observe renewals and methodological "reframings" that help establish conceptualizations of new relationships with the Other. Certain authors focus their explorations on cultural and social practices in the experience of the refugee and the displaced person as well as the development of local, national and transnational networks (Monsutti, Dicum, MosqueraLabbé, and Morissette). Others concentrate more on the symbolic and emotional dimensions of the feeling of belonging with reference to the transmission of the migrant memory, the identity project, and intergenerational transmission, leading to practices of cultural mediation (Meintel and Kahn, Dorais, Boucher, and Guzin-Lukic). Still others examine links with situations that are more on the margins of migratory 
phenomena, as they are ordinarily understood. An analysis of the identity dynamics and feeling of belonging of young professionals in the civil service of an officially multicultural and multilingual state enriches the consideration of similar phenomena in situations of migration (Veillette). Tourist trips involving a "return" to one's country of origin (Fourcade) and long-term voyages combining leisure and work in a form of modern nomadic life, "the full-time RVer" (Forget), illustrate a few forms of mobility that remain to be fully defined.

Alessandro Monsutti's article triggers an epistemological reflection on field methodologies and investigation methods. Instead of settling upon a fixed place of observation, in situ observation, the ethnologist will instead follow the movement of individuals and groups in their peregrinations. In order to carry out the study of networks, the ethnologist will no longer be content to reproduce relations through graphs, but instead will follow the actual development of the networks. Hence, the ethnologist becomes a migrant himself. Monsutti uses a network-study methodology that is renewed and articulated around interpersonal relations and displacements as a means of examining cultural and social practices, as well as strategies of economic diversification and of survival through spatial dispersal. As a result, the distinctions between the categories of refugee, displaced person, voluntary migrant, and economic migrant are highly qualified here, for a large proportion of this Hazara population exhibits, simultaneously or successively, traits of all these categories. These refugees are not merely simple victims of a fate that has overtaken them, Monsutti contends; they know how to use their cultural resources to adapt to dramatic circumstances; they develop a certain number of social strategies resembling those of economic migrants; and a given individual may belong to a number of categories at one and the same time and/or move from one to another during his or her lifetime. During this frequent back-and-forth process, "the rupture of social bonds is no longer at issue since these displacements are part of the representation of mobility that people develop to describe the course of a human life". The feeling of exile and the feeling of belonging are anchored in the representations of displacement, the meanings that are attributed to these displacements, and the use that is made of them. Alessandro Monsutti's work on the networks of Afghan Hazaras reveals the appropriation of the refugee and migratory experience in terms of a "life principle" (2004: 185), 
and shows how migration is used as an economic strategy through networks of solidarity and mutual assistance.

The social networks that migrants forge during their migration or forced displacement within or outside their own country offer opportunities to discover both their rights and new resources enabling them to ensure that these rights will be respected through participation in various support associations. By recounting the story of fifteen displaced Afro-Colombian women, Claudia Mosquera-Labbé shows the extremely precarious situation of the displaced person who does not receive the protections afforded refugees. Moreover, Mosquera-Labbés analyses reveal the new social practices of these displaced women who discover that they have legal rights that can be claimed through the practices of associations, in this way transforming themselves into political subjects.

Migrations not only serve to oppose the various cultural and social practices of migrants but also the professional practices of social interveners and educators as they interact with displaced and/or refugee populations.

Julia Dicum presents a theoretical appraisal of education programs in crisis situations with reference to an experiment involving young Afghan refugees in Pakistani refugee camps. For these young Afghans, the refugee camp where they are staying is at one and the same time a place of acculturation and of social and political experimentation. Emergency-situation education in the cultural space of the refugee camp, in a situation of transition, has to strike a balance between community orientation and providing a space for learning and for new experiences.

An individual's feeling of belonging to a community develops in tandem with the capacity that he or she has to express personal needs, expectations and suggestions, and with the feeling of being recognized for the contribution that he or she can make to this society (Guilbert 2004, 2005a). In order to successfully integrate new arrivals, a society must establish reasonable strategies of accommodation, passageways, and effective forms of mediation. A solid mastery of the language of the host country is an essential building block for successful integration, and this mastery is acquired gradually over time. Recourse to an interpreter is a fundamental identity right and proves to be indispensable when a new arrival is being received and during the first years of settling in, diminishing in frequency as the efforts of the new arrival combined 
with reception and support measures bear fruit. The situational context in which the relationship between the new arrival and his or her social environment is facilitated by the action of a person who offers linguistic mediation varies considerably according to whether the action takes place in the semi-private, public or institutional sphere. The public sphere itself requires various styles of interpretation depending on the situation; the same criteria do not apply concerning what is expected of an interpreter in a legal context as opposed to a visit to a doctor or another social service. In fact, the duties attributed to an interpreter vary according to the contexts and cultures of intervention and the balance of power operating within them (Roy and Kapoor-Kohli 2001; Guilbert 2002a, 2002b). During both intercultural citizen mediation procedures and mutual training workshops (Guilbert 2005), we have been confronted with a lively polemic concerning what is expected of interpreters. Some interested parties, often administrators of interpreter or service banks, favoured a faithful word-for-word rendering during interactions, along with physical, cultural and experiential selfeffacement on the part of the interpreter, justifying this requirement by a rather singular understanding of the notion of neutrality. Other participants, often the refugees themselves, demanded a number of changes in the management of interpreting services. They advocated greater availability with respect to interpreter service schedules (especially 24/7 emergency services), the right to choose one's own accompanying interpreter, and the right to reject an interpreter whom one does not trust or whose very presence may trespass upon the intimacy of one's remarks. These demands reflect an understanding of the interpreter's role as going far beyond that of a word-for-word translator to become that of a cultural interpreter and mediator. Still other stakeholders, usually the interpreters, reproduced in their remarks the official discourse of their employers concerning literal renderings and the interpreter's self-effacement, whereas an observation of their practices revealed an approach marked by a sensitivity to various needs and situations; often, without actually conceptualizing it, the interpreter took on the role of go-between, acting as an intercultural mediator among messengers from different cultures.

Karine Morissette examines the migratory and professional trajectory of immigrant interpreters in the Québec City region. She identifies various profiles of interpreters, professionals in the public sphere and "spontaneous" in the private sphere, and discusses different conceptions of the role of the interpreter. Do the demographic issues of Québec 
within a predominantly Anglophone Canadian nation and the linguistic tension in the Québec Francophone context influence professional practice? Can intercultural mediation really play an effective role during professional, not to mention medical, administrative or legislative proceedings? Can the Allophone-interpreter-intervener triad really avoid experiencing a power struggle?

The feeling of belonging is part and parcel of a social and cultural logic. It is constructed with strong emotional and symbolic dimensions. The question of belonging inescapably refers back to the notion of identity. Far from currents that have conceptualized identity as an accumulation of biologically transmitted innate characteristics, today's currents, marked by the dynamics of international migrations and by the influence of phenomena related to globalization, recognize the malleability and ever-changing nature of the notion of identity. Reflexivity and pluralistic ideology intervene in this work of self definition. Deirdre Meintel and Emmanuel Kahn show that the feeling of belonging may also be part of an identity project, more conscious and voluntarist on the part of parents, more diffuse and constructivist on the part of children. Whereas the parents project their identity aspirations towards their children in the future, the children forge their identity in the present according to the opportunities and spontaneous choices that arise. When these children become parents themselves, they combine what they have acquired from their parents and the issues that concern them in the pluralistic reality of Québec society in the context of contemporary globalization. This project may therefore take the form of an intergenerational negotiation. (This dimension is also picked up in the research done by both Dorais and Boucher.) Thus a sense of ethnic belonging is the product of a feeling of identity that is constantly developing; it is no longer conceived as an innate characteristic, even though, as Meintel and Kahn point out, "in people's subjective experience, this feeling still persists". Just as Monsutti does in order to capture situations of spatial and social mobility, Meintel and Kahn also reexamine the methodology of network studies in order to reach a better understanding of society "as a set of social relations branching out from each and every individual”.

The building of a feeling of belonging to a given community is influenced by the majority-minority relationship not only in a context of migration but also in the context of federal societies promoting multicultural and multilingual policies. Josianne Veillette analyzes the 
identity construction of young Québec and Swiss workers in the federal civil services of their respective countries. Without being involved in a migratory experience, these young professionals are in a daily situation of negotiating their cultural and ethnic identity, as well as their professional identity, though their strategies and positioning with respect to their capacity for and choice of using one or the other official language according to work contexts and the balance of games of power. Institutional cultures and group dynamics at one and the same time strongly influence the development of a feeling of belonging, both as regards the migratory experience and in the working environment of a plurilingual and multicultural setting.

A society can promote among its members the development of a feeling of belonging to the community through practices of cultural mediation in public space (Lamizet 1999; Guilbert 2004). The contributions of Louis-Jacques Dorais, of Colette Boucher, and of Nada Guzin-Lukic explore various facets of cultural mediation through orality, writing and museum space.

Through use of a tool designed to raise awareness concerning cultural pluralism developed by the close collaboration of an anthropologist (Dorais 2003), and of Vietnamese elders, Dorais explains how immigrant life histories open a window on the way cultural identity is constructed and recollected. Childhood memories - the basis of a Vietnamese cultural identity that is, at one and the same time, conserved, transformed and the focus of fantasy — become a social resource to be shared with the young generation of children born in Québec of Vietnamese immigrant parents, as well as with the entire population of Québec. Dorais chooses ethnographic writing in the form of a fictional presentation of an elders' discussion over tea at Dr. Quynh's, the latter being a legendary figure from Vietnamese oral tradition. The fictional presentation recalls literary proceedings in the tradition of Boccacio, Marguerite de Navarre, and the Évangiles des Quenouilles [the Distaff Gospels]. Here, however, it serves, first and foremost, the project of the migrant players themselves. The ethnologist agrees to play the instrumental role of supporting actor, catalyst, and channel of transmission in contact with committed social players who develop their cultural initiative and bring it to fruition. This cultural mediation works. Feelings of belonging to the country of origin and to the country of immigration are not in competition or in opposition. A new pathway has opened up: the development of a feeling of belonging to the country 
of adoption is a product of the free expression and recognition of the feeling of belonging to both the country of origin and the country of adoption.

The extension of this feeling of belonging to two or several cultural and national entities is constructed in transnational space. Monsutti describes the Afghan case. Elsewhere Dorais has explored these transnational identities among the Vietnamese $(2004,1998)$. The life histories analyzed by Monsutti, Meintel, Dorais and Boucher echo this transmission of family memory and desire to share a feeling of belonging. The interview with the Québec author of Haitian origin Marie-Célie Agnant, conducted by Colette Boucher, raises questions concerning orality and the transmission of cultural values through the intermediary of oral history as embedded and celebrated in the literary tale. Literary writing operates as a form of cultural mediation through which language practices, French and Creole, the feeling of belonging to both the country of origin and the country of adoption, and intergenerational communication interpenetrate and transform one another, creating identity configurations that are innovative and open to ongoing enrichment. However, these reinterpretations and reconfigurations of feelings of belonging are forged through a struggle with oneself, if not with others. The image of the warrior evokes this spirit of struggle, courage, and integrity that must be secured at any price for oneself and one's descendants. The character of the mother (and especially the grandmother) plays the role of mediator in this transmission of cultural and linguistic heritage.

This feeling of belonging to a community is constructed in a privileged manner in public space (Lamizet 1999). The museum institution can offer a form of artistic mediation likely to foster identifications with a community and reduce the "social fracture" by producing a "social bond" (Caune 1999: 44). Initiating an extension of her work on museum mediation in the context of reconciliation after the conflicts in Bosnia-Herzegovina (Guzin-Lukic 2004, 2005), Nada Guzin-Lukic expands her reflection to include the cultural mediation of the museum within pluralistic Québec society. Museums can be places of mediation between the citizen and the city, region or country. Do old stock Québecers and those with an immigrant background react the same way to the same exhibit on the development of Québec culture? Does the political and social discourse of the Museum provide an accurate representation of the pluralistic reality of contemporary Québec? 
The search for memories and the desire for cultural transmission within one's country of adoption also inform the work of Marie-Blanche Fourcade, who describes, from various angles, the links between tourist souvenirs and the diaspora. Armenian immigrants living in Québec make more or less regular visits to their country of origin and bring back tourist souvenirs to their places of residence in Québec. This "backhome tourism" [tourisme de retour], as Fourcade calls it, presents characteristics that differentiate it from ordinary tourism. Are these objects really attached to an Armenian identity and are they destined to be used the same way as before, or are they instead substitutes for lost cultural and geographical markers? Whatever the case may be, for the buyer these objects clearly represent a means of expressing a feeling of belonging to the Armenian community, with its long history, and to the diaspora.

Does the abundance of forms of mobility in today's world endanger the capacity for forging significant social bonds and maintaining a longlasting sense of belonging? Is the flexible human being an unattached human being? The "full-time camper" lifestyle illustrates an extreme form of the search for unrestrained freedom and mobility. Célia Forget provides an analysis of a mobile way of life, full-time RVing, a form of nomadism combining adventure and freedom with North American creature comforts through the technology of mobility and communications. How do these individuals, who have left everything behind to live in their recreational vehicles and to crisscross the roads of North America, cultivate a feeling of being at home while being consistently on the move? These "new nomads" are neither immigrants, refugees nor displaced persons; their permanent mobility does not bring into question their belonging to a nation or their citizenship status. The form of temporary vacation that they adopt is of an indeterminate length, and the vacationers project themselves into an imaginary realm and a fantasy world of itinerants, gypsies and hobos without experiencing all the concrete implications of such a lifestyle. They seem to have channelled two tendencies - the culture of mobility and the desire for roots - into one North American tendency: the fantasy of unrestrained pleasure and freedom. In this context, the recreational vehicle becomes the symbol of the imaginary life of the open road and a hedonistic lifestyle. However, social bonds seem to be fraying. Family bonds still resist but only insofar as each family member accepts a reinterpretation or even an inversion of the roles of each member within the family 
group. In fact, with the exception of family connections, can full-time RVing allow the formation of truly strong social ties?

\section{Migration: an opportunity for learning}

All of these articles are informed by converging lines of force. With the current proliferation of forms of mobility comes a need to examine typologies of migration and a flexible use of these definitions in order to reach a subtle understanding of such diversified human experiences. Participant observation through researcher mobility, the life histories of the migrants themselves, the study of networks recentred on concrete relational dynamics, and the cognitive and emotional meanings that the social players attribute to them all constitute promising methodological pathways. The feeling of belonging creates an integrated social field in spite of spatial dispersal. The situations experienced during migrations provide an opportunity for learning experiences, the acquisition of knowledge and expertise, the development of skills, and the deploying of personal and social resources.

Both voluntary and forced migrations provide opportunities for new learning (Guilbert 2005a, 2005b, 2005c). Forced migration is a displacement marked by loss, suffering and grief. Paradoxically, this painful journey leads to learning experiences. Those thrown headlong into exile have acquired a wide range of education even under the most difficult of circumstances.

One possibility that has not yet been explored is that an obstacle to the integration of the individual refugee or immigrant also represents the denial of the learning and knowledge acquired during the migration process. Such learning may occur during any phase of the migratory trajectory, at the local, national or international level. These learning experiences may be of an informal nature: cultural expertise being transformed into professional expertise; the creativity of cultural responses to new situations of daily life; narrative expertise; the establishing of networks; and the search for information. The learning experiences may also be formal, organized by international or local humanitarian organizations in the form of internships, vocational training courses and/or language courses. Work experiences (interpreter, language teacher, educator, social worker's assistant, tradesperson, etc.) in the intercultural and international context of refugee camps and during all stages of the migration process should also be considered. 
The learning experiences are influenced by a number of factors, including the types of refugee camps and their location, age, gender, education level, the ethnocultural group with which the refugee identifies, and the ethnocultural group with which he or she is identified by others.

We can hope that many more comparative studies of the various forms of mobility will be produced, leading to a better understanding of the possible links between migrants' learning experiences and the impact of the recognition or non-recognition of these experiences on social integration into the host society in terms of resilience, obstacles, adaptation, factors of employability, social participation, and participation as a full-fledged citizen in the life of one's new society. 


\section{References}

Amiotte-Suchet, Laurent and Pierre Floux. 2002. "Voyage avec Pierre Centlivres, de l'Afghanistan aux communautés transnationales". Ethnographiques.org.1. [On line]. http://www.ethnographiques.org/ documents/articles/centlivres.html

Camilleri, Carmel and Geneviève Vinsonneau. 1996. Psychologie et culture: Concepts et méthodes. Paris, Armand Colin.

Caune, Jean. 1999. Pour une éthique de la médiation: Le sens des pratiques culturelles. Grenoble, Presses universitaires de Grenoble.

Centlivres, Pierre and Micheline Centlivres-Demont. 2000. "Exil et diaspora afghane en Suisse et en Europe”. Cahiers d'études sur la Méditerrannée orientale et le monde turco-iranien 30: 151-172.

Coninck, Frédéric de. 2001. L'homme flexible et ses appartenances. Paris and Montréal, L'Harmattan.

Dorais, Louis-Jacques. 2004. "La construction de l'identité". In Denise Deshaies and Diane Vincent (eds.), Discours et constructions identitaires, Québec, Les Pressses de l'Université Laval: 1-11.

2003. Un thé chez le docteur Quynh. Sept aînés vietnamiens parlent de leur culture. Québec, Association Inuksiutiit Katimajiit.

1998. Identités transnationales chez les Vietnamiens du Québec. Anthropology Department, Université Laval (unpublished report).

Droz, Yvan and Beat Sottas. 1997. "Partir ou rester? Partir et rester: Migrations des Kikuyu au Kenya”. L'Homme 142: 69-88.

Guilbert, Lucille. 2005a. "La Ville de Québec: Un laboratoire pour la mise en œuvre de médiations avec les réfugiés". Revue Études ethniques au Canada / Canadian Ethnic Studies Journal, XXXVII (3): forthcoming.

2005b. "Les expériences d'apprentissage et la construction des savoirs au cours de la migration". Tenth Congress of the International Association of Intercultural Research (ARIC), Algiers, May 1-8.

2005c, "Knowledge and learning experiences acquired by refugees". Summer Course on Refugee Issues, Centre for Refugee Studies, Toronto, June 18.

- 2004. "Médiation citoyenne interculturelle: L’accueil des réfugiés dans la région de Québec." Dans Lucille Guilbert (ed.), Médiations et francophonie interculturelle, Québec, Les Presses de l'Université Laval: 199-220. 
-_.-. 2002a. "L'interprète dans sa communauté: un pont vivant". Training session for interpreters, Québec City, Centre international des femmes.

—. 2002b. "Au cœur des identités, l'interprète". Paper presented at the ACFAS Conference, Québec City, Université Laval.

Guzin-Lukic, Nada. 2004. "Patrimoine, musée et médiation". In Lucille Guilbert (ed.), Médiations et francophonie interculturelle, Québec, Les Presses de l'Université Laval: 139-157.

- - 2004. "Les musées et la guerre en ex-Yougoslavie: Vers une muséologie de la reconciliation”. Université Laval, doctoral thesis. Lamizet, Bernard. 1999. La médiation culturelle. Paris, L'Harmattan.

Monsutti, Alessandro. 2004. Guerres et migrations: Réseaux sociaux et stratégies économiques des Hazaras d'Afghanistan. Neuchâtel and Paris, Éditions de l'Institut d'ethnologie and Éditions de la Maison des sciences de l'homme.

Mucchielli, Roger. 1980. Le travail en groupe. Issy-les-Moulineaux, Éditions ESF.

Vatz-Laaroussi, Michèle. 2001. Le familial au cour de l'immigration: Les stratégies de citoyenneté des familles immigrantes au Québec et en France. Montréal, L'Harmattan.

Vinsonneau, Geneviève. 2002. L'identité culturelle. Paris, Armand Colin. 